ARTICLE

https://doi.org/10.1038/s41467-019-13163-9

\title{
Brain age prediction using deep learning uncovers associated sequence variants
}

\author{
B.A. Jonsson (1) 1,2, G. Bjornsdottir (1) 1, T.E. Thorgeirsson (1) 1, L.M. Ellingsen (1) 2 , G. Bragi Walters (1) 1,2, \\ D.F. Gudbjartsson (10) 1,2, H. Stefansson (1) 1, K. Stefansson (1) ${ }^{1,2 \star} \&$ M.O. Ulfarsson (1) ${ }^{1,2 \star}$
}

Machine learning algorithms can be trained to estimate age from brain structural MRI. The difference between an individual's predicted and chronological age, predicted age difference (PAD), is a phenotype of relevance to aging and brain disease. Here, we present a new deep learning approach to predict brain age from a T1-weighted MRI. The method was trained on a dataset of healthy Icelanders and tested on two datasets, IXI and UK Biobank, utilizing transfer learning to improve accuracy on new sites. A genome-wide association study (GWAS) of PAD in the UK Biobank data (discovery set: $N=12378$, replication set: $N=4456)$ yielded two sequence variants, $\operatorname{rs1452628-\mathrm {T}}\left(\beta=-0.08, P=1.15 \times 10^{-9}\right)$ and rs2435204-G $\left(\beta=0.102, P=9.73 \times 10^{-12}\right)$. The former is near KCNK2 and correlates with reduced sulcal width, whereas the latter correlates with reduced white matter surface area and tags a well-known inversion at 17q21.31 ( $\mathrm{H} 2)$.

\footnotetext{
${ }^{1}$ deCODE Genetics/Amgen, Inc., 101 Reykjavik, Iceland. ${ }^{2}$ University of Iceland, 101 Reykjavik, Iceland. *email: kstefans@decode.is; mou@hi.is
} 
A geing has a significant structural impact on the brain that correlates with decreased mental and physical fitness ${ }^{1}$ and increased risk of neurodegenerative diseases such as Alzheimer's disease ${ }^{2}$ and Parkinson's disease ${ }^{3}$. Recent publications, have demonstrated that MRIs can be used to predict chronological age with reasonably good accuracy ${ }^{1,4,5}$. Such predictions provide an estimate of biological brain age in independent samples. The traditional way to perform brain age prediction is to extract features from brain MRIs followed by classification or regression analysis. This includes extracting principal components ${ }^{4}$, cortical thickness and surface curvature ${ }^{6}$, volume of gray matter (GM), white matter (WM), and cerebrospinal fluid $(\mathrm{CSF})^{7}$, and constructing a similarity matrix ${ }^{8}$. The drawback of using feature extraction methods is loss of information since the features are likely not designed explicitly for extracting information relevant to brain age. Recently, deep learning (DL) methods have garnered much interest ${ }^{9}$. These methods learn features that are important without a priori bias or hypothesis. Convolutional neural networks $(\mathrm{CNNs})^{10}$ are deep learning techniques that are especially powerful for image processing and computer vision. Previously, they have been applied to brain age prediction ${ }^{11,12}$. Notably, Cole et al. ${ }^{12}$ implemented a 3D CNN trained on T1-weighted MRIs to predict brain age and achieved promising results.

PAD (the difference between predicted brain age and chronological age) estimates the deviation from healthy ageing. Studies have shown that positive PAD correlates with measures of reduced mental and physical fitness; including weaker grip strength, poorer lung function, slower walking speed, lower fluid intelligence, higher allostatic load, and increased mortality risk ${ }^{1}$. In addition, positive $\mathrm{PAD}$ has been shown to associate with cognitive impairments $s^{5,8,13,14}$, diabetes ${ }^{15}$, traumatic brain injuries $^{8}$, schizophrenia ${ }^{16,17}$, and chronic pain ${ }^{18}$. On the other hand, a negative PAD associates with higher educational attainment ${ }^{19}$, increased physical activity ${ }^{19}$, and meditation ${ }^{20}$. Moreover, PAD has been demonstrated to be heritable $\mathrm{e}^{12,21}$ and to have a polygenic overlap with brain disorders such as schizophrenia, bipolar disorder, multiple sclerosis, and Alzheimer's disease $\mathrm{e}^{21}$. Furthermore, the high degree of genetic correlation found among psychiatric and some neurological disorders suggests that current diagnostic boundaries do not necessarily reflect underlying biol$\mathrm{ogy}^{22}$. Hence, defining a novel phenotype capturing global agerelated changes in brain structure could, via variants in the sequence of the genome that associate with these changes, provide novel biological insights.

Here we present a new brain age prediction method (Fig. 1) that uses a $3 \mathrm{D}$ CNN trained on MRIs to predict brain age. The input data are a T1-weighted image registered to Montréal Neurological Institute (MNI) space and data derived from the T1weighted image, i.e., a Jacobian map, and gray and white matter segmented images (Fig. 1). The input data also include information about the subject's sex and the type of MRI scanner. The output of the network is the predicted brain age.

As mentioned above, Cole et al. ${ }^{12}$ trained a $3 \mathrm{D} \mathrm{CNN}$ to perform brain age prediction. Our network is different in four key ways. (1) We use a significantly different architecture. While their architecture resembles a standard VGGNet architecture ${ }^{23}$ our architecture uses the recent ResNet design ${ }^{24}$. One of the drawbacks of the VGG architecture is that the vanishing gradient problem limits the potential depth of the network. In contrast, the ResNet architecture has no such depth limits. ResNets also have smoother loss surfaces ${ }^{25}$, which in turn helps speeding up convergence. (2) We add inputs to the final CNN layer to factor in information about sex and scanner. (3) Our technique is the first to use deformation information encoded in Jacobian maps to predict brain age. (4) As we have mentioned, our method combines predictions from multiple CNNs by either averaging predictions or by training a data blender.

In experiments, we compare our proposed method to a few brain age prediction methods based on feature extraction and machine learning. We also demonstrate that transfer learning is useful for adapting a CNN trained to predict brain age on one site to a new site while retaining predictive accuracy. And we look at how the PAD calculated with our method is affected by random weight initialization and retraining. We then check for associations between PAD and performance on neuropsychological tests. Finally, we perform genetic analysis on PAD using UK Biobank data, resulting in identification of associations with five sequence variants for which we provide detailed phenotypic characterizations.

\section{Results}

Combining CNN outputs improves prediction accuracy. Our brain age prediction method was developed using images from structural brain MRIs for 1264 healthy Icelanders. To overcome problems caused by training a DL method on such a small dataset we use multiple images of the same individuals and utilize a data augmentation strategy. We start off by training the method independently on the four previously mentioned image types (Table 1A). The CNN that predicts the test set with the least error is the CNN trained on T1-weighted images followed by the CNN trained on WM segmented images (Supplementary Figs. 4 and 5 show scatter plots of the CNN test set predictions against chronological age).

Having four predictions from four different data sources opens up the possibility of combining the predictions. The most straightforward way of fusing the forecasts is by using a majority voting scheme, e.g., by averaging the predictions made by the four CNNs. Another way to combine forecasts is to implement a data blender, for example, by implementing a linear regression model trained to predict brain age from the four CNN brain age predictions. This technique attempts to find the best linear combination of the four brain age predictions so in theory it should be guaranteed to be at least as good as the best predicting $\mathrm{CNN}$ method. To demonstrate this, we tried combining CNN brain age predictions using majority voting and linear regression data blending (Table 1B). Comparing the test set results of Table $1 \mathrm{~B}$ to the results in Table $1 \mathrm{~A}$, we see that combining predictions results in lower test error than achieved by the CNN trained on $\mathrm{T} 1$-weighted images.

It is not straightforward to compare the accuracy of our method to previous brain age prediction methods, because they are evaluated on other datasets. However, to establish a baseline for the CNN-based techniques, we investigated methods based on feature extraction such as surface-based morphometry $(\mathrm{SBM})^{26}$, voxel-based morphometry $(\mathrm{VBM})^{27}$, and similarity matrices. Machine learning regression methods were trained on these three types of features separately. For each feature type, we trained eight different types of regression methods. The list of methods we tried is far from exhaustive, instead these methods were chosen to represent commonly used and relatively simple to tune regression methods. In addition, we considered methods such as relevance vector regression $(\mathrm{RVR})^{28}$ and Gaussian process regression (GPR $)^{29}$ which have previously been successfully used to predict brain age ${ }^{4,8}$. Table $1 \mathrm{C}$ shows the prediction results for the regression models with the lowest test error for each feature type (the Methods section and Supplementary Table 1 include more information and results for the regression methods trained on these features). In addition, Table $1 \mathrm{C}$ shows results for combining the best predictions for SBM, VBM and similarity matrix features using the same methods used to combine the 


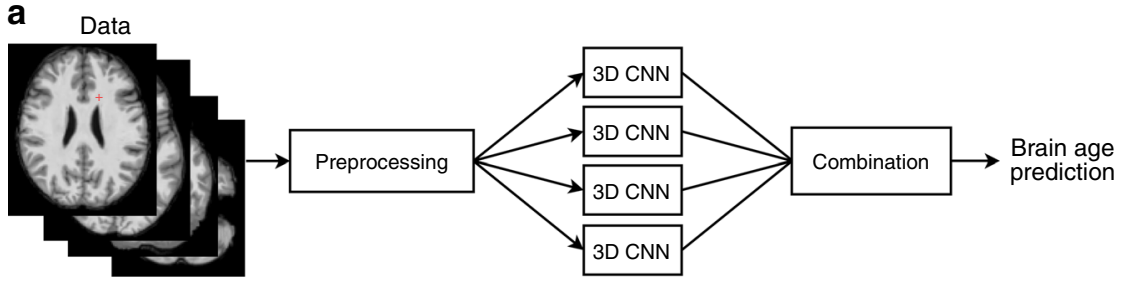

b

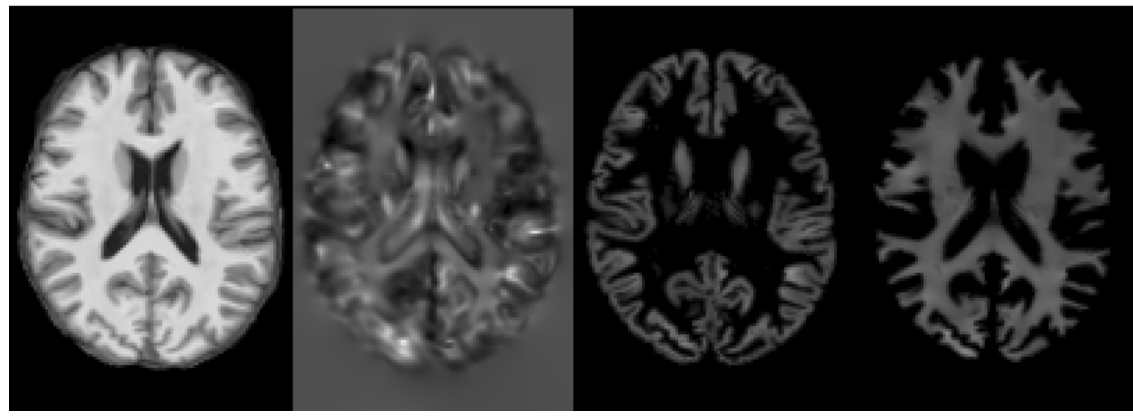

Fig. 1 Illustration of the proposed method and input data. a A flowchart showing a high-level overview of the proposed brain age prediction system. b Examples of image types generated by the preprocessing step. From left to right: a registered T1-weighted slice, a Jacobian map slice, a GM segmented slice, and a WM segmented slice.

\begin{tabular}{|c|c|c|c|c|c|c|c|}
\hline & Type & Method & Val MAE & Val $R^{2}$ & Test MAE & Test $R^{2}$ & No. I \\
\hline \multirow[t]{3}{*}{ (A) } & T1-weighted & CNN & 3.996 & 0.810 & 4.006 & 0.829 & 1815 \\
\hline & Gray matter & CNN & 4.766 & 0.721 & 4.641 & 0.776 & 1815 \\
\hline & White matter & CNN & 4.676 & 0.735 & 4.189 & 0.812 & 1815 \\
\hline \multirow[t]{3}{*}{ (B) } & $\mathrm{MV}(\mathrm{T} 1$ and $\mathrm{JM})$ & CNN & 4.102 & 0.803 & 3.919 & 0.841 & 1815 \\
\hline & $\mathrm{MV}(\mathrm{T} 1, \mathrm{JM}, \mathrm{GM}$, and $\mathrm{WM})$ & CNN & 3.845 & 0.849 & 3.584 & 0.849 & 1815 \\
\hline & LRB (T1, JM, GM, and WM) & CNN & 3.581 & 0.847 & 3.388 & 0.872 & 1815 \\
\hline \multirow[t]{4}{*}{ (C) } & SBM & $\mathrm{RR}$ & 5.268 & 0.689 & 5.176 & 0.697 & 1320 \\
\hline & VBM & GPR & 4.278 & 0.781 & 4.317 & 0.766 & 1794 \\
\hline & SM & $\mathrm{RR}$ & 4.898 & 0.722 & 4.937 & 0.728 & 1815 \\
\hline & MV (SBM, VBM, and SM) & GPR/RR & 4.008 & 0.808 & 3.940 & 0.761 & 1246 \\
\hline
\end{tabular}

CNN predictions. Similarly to the CNNs, the combined predictions have lower test MAE than any of the methods limited to single feature types. However, if we compare the results in Table $1 \mathrm{~B}$ and $\mathrm{C}$ we see that the predictions made with combined $\mathrm{CNN}$ outputs are more accurate than any of those based on the feature extraction methods.

Testing the CNN on other datasets. Next, we examine how the method performs if we predict brain age of images from other datasets. To do so, we evaluate it on the IXI and UK Biobank ${ }^{30}$ datasets and combine predictions using majority voting. We use this combination method rather than data blending because it has similar accuracy to linear regression blender with the added benefit that it is unnecessary to train an extra linear model on the predictions. We observe that the initial prediction error of the method is high (Table 2). The problem is that there can be subtle differences between data from different scanning sites which will cause a model trained on one site to fail when predicting on the other site. There are multiple reasons for this. The MRI scanner type and parameters between sites can be different, which can cause differences between resolution, contrast and noise levels. Also, the distribution of age can be different between sites, for example, it is problematic if the new site has a wider age range than the training set.

We hypothesize that a CNN that is already proficient at predicting brain age at one site only needs a small adjustment to adapt to data from a new site. A transfer learning strategy achieves this: First, we freeze the model weights of the convolutional layers so that only the fully connected layers are trainable. Second, the CNN is re-trained on a portion of the data from the new site. An advantage of this strategy is that there are 
Table 2 UK Biobank and IXI prediction performance with and without transfer learning.

\begin{tabular}{|c|c|c|c|c|c|c|}
\hline & IXI & & & UK Biobanl & & \\
\hline TL Used & Val MAE & Val $R^{2}$ & Val set size & Test MAE & Test $R^{2}$ & Test set size \\
\hline No & 6.420 & 0.778 & 104 & 8.494 & -0.630 & 12395 \\
\hline Yes & 4.149 & 0.907 & 104 & 3.631 & 0.614 & 12395 \\
\hline
\end{tabular}

now fewer parameters to train, which means we can use less data and training will be faster. We carry out the transfer learning strategy by retraining the majority voting $\mathrm{CNN}$ on 440 images from the IXI dataset.

The re-trained $\mathrm{CNN}$ is validated on 104 images from the IXI dataset left out during training (validation set) and tested on 12395 images from the UK Biobank dataset (test set). Table 2 shows that the prediction accuracy is increased significantly by doing so. In addition, the test set predictions before and after transfer learning are shown on a scatter plot against chronological age (Supplementary Fig. 6). Surprisingly the accuracy of predictions for the UK Biobank site improve even though the CNN was not explicitly trained on it. This is intriguing and is perhaps explained by the fact that the IXI set includes a wider age range than the Icelandic set and includes 3T MRI images unlike the Icelandic set.

In subsequent sections, the CNNs trained with transfer learning on the IXI sample will be used in downstream analysis of the UK Biobank sample. While it is likely that transfer learning on a small subset of the UK Biobank sample will lower the UK Biobank test MAE, we refrained from doing this because we want to use as many subjects as possible in the downstream analysis and because of the limited age range in the UK Biobank sample (all subjects are in the age range $45-80$ years). Training on such a limited age range would severely bias the model towards predicting ages inside this range. To get around this, it is necessary to train the model on a sample with a wider age range. This is why we use the CNNs trained on the IXI sample, which includes subjects in the age range $20-86$ years, in the downstream analysis.

Effect of random CNN weight initialization on PAD. We know that because CNNs start out in random initial states, and because they have highly non-convex loss functions ${ }^{25}$, it is possible that two randomly initialized instances of our brain age prediction method will converge to two distinct local minima. These states could in theory both predict age equally well but have uncorrelated PAD values. Here we face a potential problem, because in the absence of a ground truth for the PAD there is no way to tell if either one of these PAD predictions is accurate. This sort of unreliable CNN behavior would be problematic for any downstream analysis that utilizes the brain age prediction, because any conclusions made about the PAD would depend on the initialization of the CNN. In light of this, it would be reassuring if we could demonstrate that our method generally converges to similar $\mathrm{PAD}$ predictions after training.

To test this, four additional randomly initialized instances of our brain age prediction method are trained and the agreement between their PADs is examined. This procedure entails repeating these three main steps four times: (1) Train four CNNs on the Icelandic dataset on the four previously mentioned image types. (2) Freeze convolutions layers and train the CNNs on the IXI dataset (transfer learning step). (3) Predict brain age in the UK Biobank dataset using CNNs, combine the predictions with majority voting and calculate $\mathrm{PAD}$ values.
After repeating these steps, we get four instances of the brain age prediction method that predict brain age of the 12395 subjects in the UK Biobank with mean absolute error (MAE) equal to 4.6, $5.5,5.4$, and 4.9 years, respectively. The reason why the error is higher here compared with the original results is that we did not reinitialize and retrain the CNNs in cases were the optimization got stuck in a poor local minimum or a saddle point. Nevertheless, if we look at the agreement of the original and the four new PADs we find that the intraclass correlation (ICC) is estimated to be equal to 0.86 (95\% confidence interval $[\mathrm{CI}]=$ $[0.855,0.863])$. This indicates that the UK Biobank PAD calculated using our method stays rather consistent between the five different training runs and is relatively robust to random weight initialization.

Associations between PAD and performance on neuropsychological tests. As mentioned above, previous studies have linked high PAD to cognitive impairment $5,8,13,14$. In light of this, we are interested in looking at if PAD associates with performance on neuropsychological tests. Specifically, performance on tests administered by the UK Biobank that are designed to measure: fluid intelligence, numeric memory, visual memory, prospective memory, simple processing speed, complex processing speed, visual attention, and verbal fluency. To estimate PAD in the UK Biobank, we train four CNNs on the Icelandic set, then the IXI set using transfer learning, and combine their predictions using majority voting. We did not find evidence of association between PAD and performance on the fluid intelligence, numeric memory, pairs matching, and prospective memory tests (Supplementary Table 2 includes these results). However, we see from Table 3 that PAD is associated with worse performance on the digit substitution test (DSST), trail making tests (TMTs), and the reaction time test (a more detailed description of the tests can be found in Supplementary Notes 1-7). As expected, these results indicate that PAD is in fact associated with cognitive impairment.

Genome-wide association study. PAD has previously been shown to be heritable ${ }^{12,21}$, however, to our knowledge no sequence variants conferring risk of or protecting against PAD have been identified. In order to look for such variants, we ran a genome wide association scan (GWAS) in the UK Biobank sample on PAD (same PAD as from the section Testing the CNN on other datasets) using BOLT-LMM ${ }^{31}$. This scan yields two sequence variants, rs2435204-G and rs1452628-T (Fig. 2 and Table 4A) (Supplementary Figs. 8 and 9 show locus zoom plots for the two genome-wide significant variants). In addition, given that sequence variants known to associate with brain structure are likely to be enriched for variants that associate with PAD. We decided to test a smaller set of 331 brain structure variants for association with PAD. This yielded associations with three additional variants (Table 4B). For more information, the 'Statistical methods' section contains information about how the brain structure variants were identified.

The high number of tests conducted in GWAS combined with the general small effect size of common markers greatly 


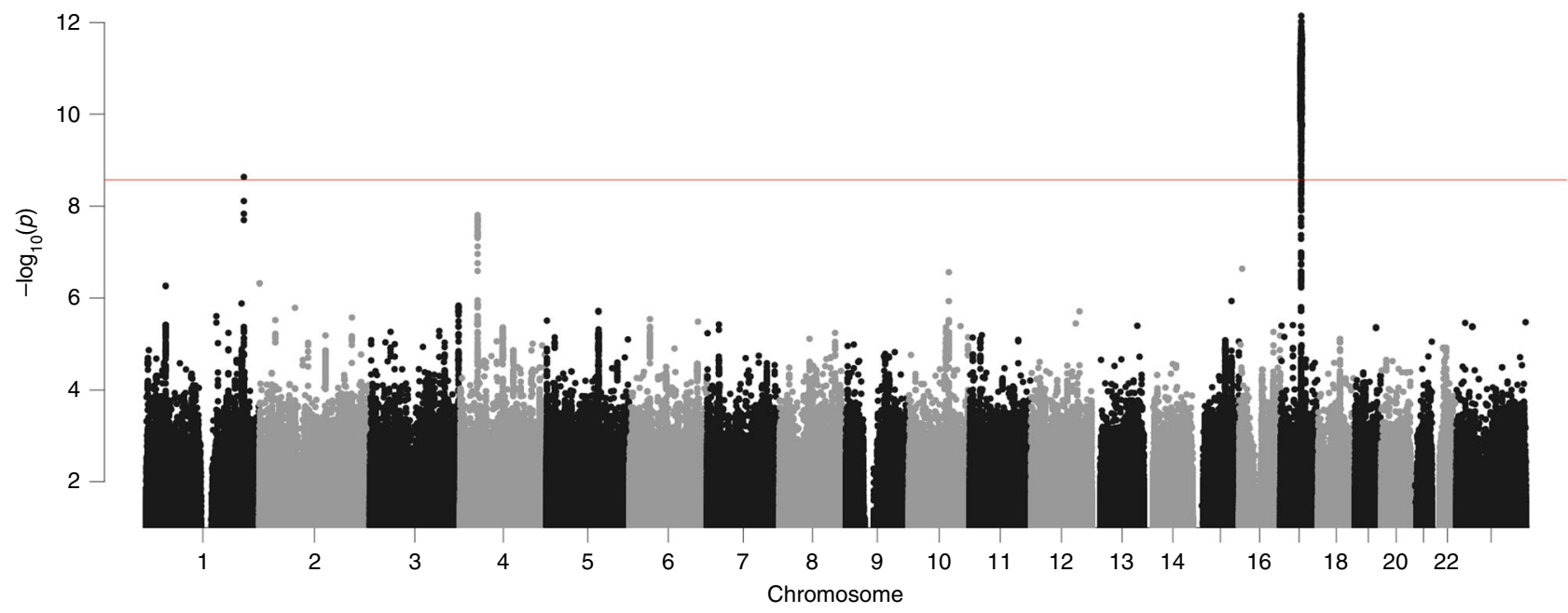

Fig. 2 Manhattan plot of the GWAS results for the UK Biobank data. The horizontal line denotes the $P$ value threshold for genome-wide significant effect.

Table 3 Pearson's $r$ correlation between PAD and performance on neuropsychological tests.

\begin{tabular}{|c|c|c|c|c|}
\hline Neuropsychological test & PAD correlation & $95 \% \mathrm{Cl}$ & $P$ Value & No. subjects \\
\hline DSST & -0.080 & $(-0.104,-0.054)$ & $4.3 e-11$ & 6849 \\
\hline TMT B & 0.076 & $(0.051,0.103)$ & $3.1 e-09$ & 6076 \\
\hline TMT A & 0.053 & $(0.027,0.078)$ & $3.8 e-05$ & 6076 \\
\hline Reaction time & 0.030 & $(0.012,0.047)$ & $7.9 e-04$ & 12387 \\
\hline
\end{tabular}

Table 4 Sequence variants associated with PAD estimated using BOLT-LMM.

\begin{tabular}{|c|c|c|c|c|c|c|c|}
\hline (A) & rs1452628 & chr1:214966544 & $\mathrm{T} / \mathrm{A}$ & 36.2 & -0.08 & $(-0.10,-0.05)$ & $2.3 e-09$ \\
\hline \multirow{2}{*}{ (B) } & rs6437412 & chr3:194747684 & $\mathrm{C} / \mathrm{T}$ & 28.2 & -0.06 & $(-0.09,-0.04)$ & $6.8 e-06$ \\
\hline & rs2184968 & chr6:126439848 & $\mathrm{C} / \mathrm{T}$ & 46.0 & 0.05 & $(0.03,0.08)$ & $7.5 e-05$ \\
\hline \multirow[t]{2}{*}{ (C) } & rs2435204 & chr17:45910839 & $G / A$ & 26.6 & 0.08 & $(0.03,0.13)$ & $1.5 e-03$ \\
\hline & rs1452628 & chr1:214966544 & $T / A$ & 36.2 & -0.07 & $(-0.12,-0.03)$ & $8.8 e-04$ \\
\hline (D) & rs2184968 & chr6:126439848 & $\mathrm{C} / \mathrm{T}$ & 46.0 & 0.06 & $(0.02,0.10)$ & $2.9 e-03$ \\
\hline
\end{tabular}

$(A, B)$ Association between sequence variants and PAD for 12378 subjects in discovery set. (A) Genome-wide significant sequence variants. (B) Sequence variants associated with structural MRI brain phenotypes that also associate with PAD. (C, D) Association between sequence variants and PAD for 4456 subjects from the replication set. (C) Genome-wide significant sequence variants. (D) Sequence variants associated with structural MRI brain phenotypes that also associate with PAD. Note that the reported effect sizes are for PAD normalized to unit variance. Before normalization the standard deviation of PAD was $\sim 4$ years. Thus the associated lowering of the protective allele of rs 1452628 is approximately -0.32 years

standard deviation of PAD was $\sim 4$ years. Thus the
$\mathrm{Cl}$ confidence interval, MAF minor allele frequency

increases the risk of a false postives ${ }^{32}$. To protect against potential confound effects we adjusted for potential nuisance variables, such as age, gender, total intracranial volume, principal components from genetic ancestry analysis, head motion, genotyping array, and imaging center. In addition, we removed individuals of non-white British ancestry and one subject from each related pair of individuals (the Statistical Methods section provides more information about the exact adjustment procedure). And then to thoroughly vet each hit we took two steps. (1) We performed a replication test on held out data. (2) Checked if the reported variants associate with other phenotypes related to brain ageing.

(1) The five reported sequence variants also associated with PAD in a replication set of 4456 subjects (Table 4 [C, D]). Four other variants which came up in the discovery stage were omitted because they did not replicate. (2) The identified sequence variants also associate with brain structure likely to be affected by brain ageing. Associations between these sequence variants and SBM/VBM brain structure phenotypes and correlation between $\mathrm{PAD}$ and the brain structure phenotypes are shown in Supplementary Tables 4 and 5. Supplementary Table 4 shows that both PAD and rs1452628-T associate with lower CSF throughout the cerebral cortex which is consistent with reduced cortical sulcal openings. On the other hand, rs2435204-G associates with lower total white matter surface area, and reduced area in a number of cortical brain regions (Supplementary Table 5). Although it was known a priori that the other three sequence variants would associate with structural brain 
phenotypes, the specific structural brain phenotypes that associate with these variants are listed in Supplementary Tables 6-8.

Running LD score regression ${ }^{33}$ on the GWAS results, we estimated the SNP-heritability for PAD to be $h_{\text {snp }}^{2}=0.264(95 \%$ $\mathrm{CI}=[0.178,0.350])$. In addition, the intercept of the LD score regression model is equal to $0.991(95 \% \mathrm{CI}=[0.979,1.003])$, which indicates that the model did not find any evidence of confounding effects in the PAD GWAS results. This $h_{\text {snp }}^{2}$ estimate is close to the one previously estimated by Kaufmann et al. ${ }^{21}$ $\left(h_{\mathrm{snp}}^{2}=0.1828[\mathrm{SE}=0.02]\right)$. And predictably our $h_{\mathrm{snp}}^{2}$ is lower than the narrow-sense heritability estimate of PAD estimated by Cole et al. ${ }^{12}\left(h^{2}=0.66[\mathrm{SE}=0.09]\right)$ using a twin study sample.

\section{Discussion}

Here, we have presented a novel deep learning approach, using residual convolutional neural networks to predict brain age from a T1-weighted MRI, a Jacobian map, and gray and white matter segmented images, to study the discrepancy between agerelated structural brain changes and chronological age. The MRI based deep learning system was shown to predict brain age from T1-weighted MRI data with a $\mathrm{MAE}=3.39$ and $R^{2}=0.87$ on test data. Comparing our approach to other machine learning methods trained on surface-based morphometry, voxel-based morphometry, and similarity matrix features, we showed that our approach predicts brain age more accurately. We showed that transfer learning can be used to efficiently increase prediction accuracy for new sites. The PAD calculated using this method was shown to be relatively robust to random weight initialization and retraining, a result that indicates that the PAD estimated using our method can be used as a reliable phenotype in the study of brain ageing, as well as in the study of specific disorders of the brain. We also proposed that PAD could be an informative phenotype for genetic association studies, and indeed, our association analysis of PAD in a discovery set of 12378 subjects and replication set of 4456 subjects yielded five sequence variants.

The sequence variant with the strongest association, rs2435204-G, tags the $\mathrm{H} 2$ (inverted) form of the 17q21.31 inversion polymorphism ${ }^{34}$. This inversion spans $\sim 1 \mathrm{Mb}$ and includes 10 genes, including MAPT, a gene that encodes the tau protein which has been implicated in various dementias ${ }^{35}$. In addition, micro-deletions within the inversion are known to cause intellectual disability ${ }^{36}$. The $\mathrm{H} 1$ inversion haplotype has been associated with increased risk of Parkinson's disease, malepattern baldness, and several other phenotypes, whereas $\mathrm{H} 2$ has been associated with a number of phenotypes including neuroticism $^{37}$, fibromyalgia ${ }^{18}$, lower educational attainment, increased fecundity $^{38}$, and smaller intracranial volume (ICV) ${ }^{39}$ (Note that PAD is adjusted for ICV, thus the observed effect on PAD is not caused by ICV). Due to the extensive linkage disequilibrium (LD) the $17 \mathrm{q} 21.31$ inversion region, reported markers for various associations in the region often differ between studies. For example, the most recent GWAS meta-analysis of Parkinson's disease reports an association with rs17649553-T, that is fixated on and highly correlated with the H2-tagging rs2435204-G $\left(r^{2}=0.82, D^{\prime}=1\right)$, with OR $=0.78(95 \% \mathrm{CI}=[0.76,0.80]), P=$ $1.26 \times 10^{-68}$ (their meta-analysis was carried out with a fixedeffects model based on inverse-variance weighting) ${ }^{40}$.

rs2435204-G also associates with brain structure phenotypes. Supplementary Table 5, shows that both PAD and rs2435204-G associate with increased thickness and decreased area in cortical brain regions. Interestingly, this pattern of increased thickness and decreased area has previously been associated with neuroticism $^{41}$. Thus, lifestyle or phenotypes associated with a high neuroticism score, including anxiety, worry, fear, anger, frustration, depressed mood, and loneliness may associate with PAD.

The other genome-wide significant sequence variant, rs1452628$\mathrm{T}$, is located close to KCNK2 (also known as TREK1), which belongs to the two-pore domain potassium channel family and is mainly expressed in the brain ${ }^{42}$. In mice, KCNK2 has been implicated in neuroinflammation ${ }^{43}$, cerebral ischemia ${ }^{44}$, and blood-brain barrier dysfunction $^{45}$. rs1452628-T correlates with SNPs that have previously been associated with cortical sulcal opening and GM thickness, rs6667184 $\left(r^{2}=0.68\right)$, and rs864736 $\left(r^{2}=0.49\right)^{46}$.

In addition, we identified three sequence variants associated with PAD by restricting the analysis to SNPs known a priori to associate with structural phenotypes. (1) rs2790099-C is located in an intron of $R U N X 2$, a gene that encodes the RUNX2 protein which is essential for osteoblastic differentiation and skeletal morphogenesis and has been shown to play several roles in cell cycle regulation ${ }^{47}$. Supplementary Fig. 7 shows that rs2790099-C is a possible cis-eQTL of RUNX2 and it is most expressed in the basal ganglia (caudate and putamen). This lines up with the a priori brain structure GWAS that shows that rs2790099-C has genome-wide significant associations with white matter volume of regions in the basal ganglia (putamen and pallidum) (Supplementary Table 6). (2) rs6437412-C is an intron variant of LINC01968 that associates with increased cortical CSF (Supplementary Table 7). (3) rs2184968-C is located in an intron of $C E N P W$, a gene that has previously been associated with traits, such as, height ${ }^{48}$, cognitive performance ${ }^{49}$, and male-pattern baldness $^{50}$. Our analysis shows that rs2184968-C is associated with increased CSF in subcortical regions and increased size of the fourth ventricle (Supplementary Table 8).

Confound effects are a problem for big imaging studies due to the huge number of imaging artifacts that can potentially influence both imaging and non-imaging variables of interest ${ }^{32}$. Some of the confound effects we have tried to control for are effects due to age, sex, head size, population structure, and scanner type. Head motion is another potentially problematic confound effect, because it causes reduction of estimated gray matter volume and thickness in MRI images similar to what we expect to see due to ageing ${ }^{51}$. While head motion is not important in the evaluation of our method (see Cole et al. ${ }^{12}$ ), it is potentially a problematic confound for GWAS analysis because certain clinical groups associate more with scanner motion. Elliott et al. ${ }^{52}$ suggest to use fMRI-derived head motion estimates to correct for confound effects due to head motion when running GWAS analysis on brain structure phenotypes. We adjusted PAD for head motion as they suggest, however, this correction only had a small effect on our results. Other potential confounds that we looked at were sample relatedness (the first 40 principal from components genetic ancestry analysis), genotyping array, and the assessment center where neuropsychological testing was performed. As with head motion, adjusting for these variables did not affect our results.

From our analysis we see that PAD associates with worse performance on neuropsychological tests, specifically poor performance on DSST, TMT, and the reaction time tests (Table 3). Interestingly, both the DSST and the reaction time test are designed to measure cognitive processing speed. The TMT is designed to asses visual attention. However, psychomotor speed is a factor in successful TMT performance ${ }^{53}$. Furthermore, a decline in processing speed along with impairment of reasoning, memory, and executive function are well documented to occur in ageassociated cognitive decline ${ }^{54}$. As such, these results are in line with other studies that link high PAD to cognitive impairment ${ }^{5,8,13,14}$. We note, that the association between PAD and TMT is consistent with the previous finding of Cole et al. ${ }^{8}$. However, the large dataset used here gives more conclusive results. Supporting this, we additionally find that schizophrenia, a 
brain disorder characterized by complex patterns of cognitive impairment, correlates with positive PAD (greater brain ageing than chronological age) and (Supplementary Table 3).

In conclusion, we have presented a new method for predicting brain age using cutting-edge machine learning techniques. Our deep learning method produces a single measure (PAD) from raw MRI data that captures complex underlying correlated changes in MRI and can be used to study various traits and diseases, and in particular for genetic discovery. Using such a method represents one potential way for overcoming challenges with high dimensional data and multiple testing that plagues MRI research. Applying our method to large genomic datasets such as the UK Biobank has enabled us to identify novel associations between sequence variants and brain ageing. The variants identified are common SNPs with small effects on PAD (Table 4) accounting for only a fraction of the trait variance. However, these first findings provide a foothold, and further research into these loci as well as additional GWAS studies have potential to shed light on the biological underpinnings of the ageing brain and its connection to various diseases and disorders.

\section{Methods}

Datasets. The proposed method was evaluated on T1-weighted MR images from three independent datasets: an Icelandic dataset, the UK Biobank dataset, and the IXI dataset. DeCODE genetics provided the Icelandic MR data, consisting of scans from 1264 healthy subjects aged between 18 and 75 years. This dataset includes 1815 scans in total, since some subjects have several scans. The Icelandic data were acquired using two different scanners, a 1.5T Phillips Achieva scanner, and a $1.5 \mathrm{~T}$ Siemens Magnetom Aera scanner. Scans were imaged using a T1-weighted gradient echo sequence (Philips Achieva: repetition time $(\mathrm{TR})=8.6 \mathrm{~ms}$, echo time $(\mathrm{TE})=$ $4.0 \mathrm{~ms}$, flip angle $(\mathrm{FA})=8^{\circ}, 170$ slices, slice thickness $=1.2 \mathrm{~mm}$, acquisition matrix $=192 \times 192$, FOV $=240 \times 240 \mathrm{~mm}$; Siemens Aera: repetition time $(\mathrm{TR})=$ $2400 \mathrm{~ms}$, echo time $(\mathrm{TE})=3.54 \mathrm{~ms}$, flip angle $(\mathrm{FA})=8^{\circ}, 160$ slices, slice thickness $=1.2 \mathrm{~mm}$, acquisition matrix $=192 \times 192$, FOV $=240 \times 240 \mathrm{~mm}$ ). Any serious neurological disorders were prescreened and removed. In addition, we removed from the training and holdout sets subjects diagnosed with neurodevelopmental and mental disorders such as autism, bipolar disorder, intellectual disability, or schizophrenia, and subjects with any copy number variations previously associated with neurodevelopmental or psychiatric disorders.

The UK Biobank dataset consists of T1-weighted MR images of 15040 healthy subjects aged between 46 and 79 years old. The data were all collected using a $3 \mathrm{~T}$ Siemens Skyra scanner. It is well-known that the presence of undetected population structure can lead to both false positive results and failure to detect genuine associations in genetic association studies ${ }^{55}$, in an effort to combat this our analysis was constrained to 12378 individuals of white British ancestry. An additional release of MRI images by UK Biobank was added to a replication set. This set contains 6888 subjects (thereof 4456 subjects of white British ancestry) aged between 47 and 80 years old. The images in this set were collected using the same protocol as the previous UK Biobank set.

The IXI dataset consists of T1-weighted MR images of 544 healthy subjects and is freely available online. The subjects age at imaging was between 20 and 86 years old. The IXI data were collected from three different sites. The Hammersmith Hospital using a Philips 3T system, Guy's Hospital using a Philips 1.5T system and the Institute of Psychiatry using a GE 1.5T system. Histograms of the age distribution of the three datasets mentioned are shown in Supplementary Figs. 1-3.

Preprocessing. Preprocessing was carried out using the computational anatomy toolbox (CAT12) ${ }^{56}$. First, the input data were inhomogeneity corrected. Then the skull and other non-brain elements were removed. Finally, the images were registered into the standard MNI space using the deformable registration algorithm DARTEL $^{57}$. For further information, refer to the CAT12 manual ${ }^{58}$.

There are three types of images that the preprocessing step generates. The first is an MNI-registered image. Second, a Jacobian map which is a by-product of the deformable registration. Last, a gray matter and white matter soft segmented image. All of the image types mentioned above have voxel size $1.5 \mathrm{~mm}^{3}$ and voxel resolution $121 \times 145 \times 121$.

CNN architecture. The CNN architecture we developed is based on the residual architecture $^{24}$ (Fig. 3). It was implemented using Keras with TensorFlow as backend ${ }^{59}$ and consists of five residual blocks, each followed by a max pooling layer of stride $2 \times 2 \times 2$ and kernel size $3 \times 3 \times 3$, and one fully connected block. The convolutional part of the $\mathrm{CNN}$ reduces the input image from size $121 \times 145 \times 121$ to 128 feature maps of size $4 \times 5 \times 4$. The fully connected part reduces these feature maps down to an age prediction.

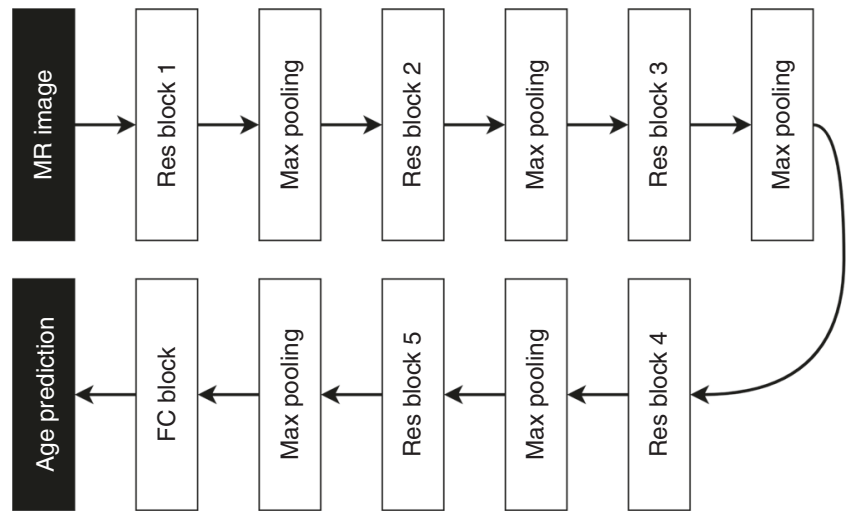

Fig. 3 A flowchart showing the components of the proposed CNN architecture. Residual (Res), fully connected (FC).

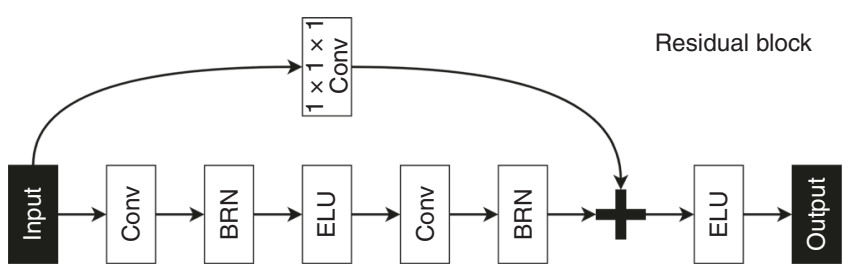

Fig. $4 \mathrm{~A}$ flowchart showing the components of the proposed residual block. Batch re-normalization (BRN), convolutional layer (Conv).

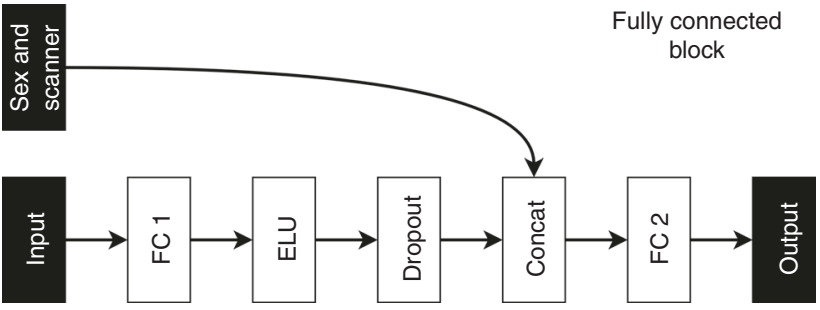

Fig. 5 A flowchart showing the components of the proposed fully connected block. Fully connected layer one (FC1), concatenation layer (Concat), fully connected layer two (FC2).

The residual block, displayed in Fig. 4, consists of a combination of layers which are repeated twice inside the residual blocks. This combination is composed of a 3D convolutional layer with stride $1 \times 1 \times 1$ and kernel size $3 \times 3 \times 3$, a batch renormalization layer ${ }^{60}$, and an ELU activation function ${ }^{61}$. The defining element of the residual block is the skip connection which adds the signal feeding into the residual block to the output of a layer close to the end of the block. The number of feature maps in block number $n$ was chosen by the rule $2^{n+2}$.

The fully connected block, depicted in Fig. 5, is a multilayer perceptron (MLP) ${ }^{62}$ with one hidden layer. The input layer has $128 \times 4 \times 5 \times 4=10240$ neurons, the hidden layer (FC 1) has 256 neurons that use an ELU activation function, and the output layer has a single neuron. Following the hidden layer, a dropout ${ }^{63}$ layer with keep rate equal to 0.8 is employed. The output layer (FC 2) has no activation function which means that it performs a linear regression on the hidden layer features. To account for factors such as scanner type and sex that can affect the estimated brain age of an individual we include them as inputs in the linear regression by concatenating them with the hidden features of the MLP.

The mean absolute error was used as the loss function and the CNN was optimized using Adam ${ }^{64}$ with parameters: learning rate $=0.001$, decay $=10^{-6}$ $\beta_{1}=0.9, \beta_{2}=0.999$, and batch size $=4$. The He initialization strategy ${ }^{65}$ was used to initialize the weights, and each trainable node in the CNN was regularized with $l_{2}$ weight decay ${ }^{66}$, with $\lambda=5 \times 10^{-5}$. Early stopping ${ }^{67}$ with model

checkpointing was used, i.e., if the validation error did not improve in 100 epochs the training was stopped and the weights with the lowest validation error selected. Furthermore, to reduce the risk of overfitting, data augmentation ${ }^{68}$ was used to generate new training instances by applying a coordinate transformation to a random subset of the training data, consisting of a combined $3 \mathrm{D}$ rotation and a $3 \mathrm{D}$ translation. The rotation angles were between $-40^{\circ}$ and $40^{\circ}$ with equal probability, 
and the translation distance, for each direction, was selected between -10 and 10 voxels with equal probability.

Our CNN implementation uses about $\sim 8 \mathrm{~GB}$ of memory and the training time using an Intel Xeon Gold 6130 Processor CPU with $32 \mathrm{~GB}$ of RAM and an NVIDIA Tesla V100 16GB GPU was about 2 days.

SBM, VBM, and similarity matrix brain age prediction. The SBM features were generated using FreeSurfer's recon-all algorithm ${ }^{69}$ and the VBM features were generated using the CAT12 toolbox (the specific names of the SBM and VBM features are listed in Supplementary Data 1). The similarity matrix was constructed by taking the inner product between the combined gray and white matter segmented images of each subject. The SBM and VBM features were adjusted for intracranial volume, sex and scanner type. The features were then zero centered and normalized to unit variance. The regression methods that were tested were, linear regression ${ }^{70}$, lasso ${ }^{71}$, ridge regression ${ }^{72}$, elastic net ${ }^{73}$, random forest regres$\operatorname{sion}^{74}$, support vector regression ${ }^{75}$, relevance vector regression ${ }^{28}$, and Gaussian process regression ${ }^{29}$. A grid search was used to find the tuning parameters corresponding to the lowest cross-validation error for the methods mentioned. The regression models were implemented using scikit-learn ${ }^{76}$, except relevance vector machines, which used scikit-rvm.

In addition, we tested combining predictions made by models trained on these three feature types. We decided to pick predictions for the method with the lowest CV MAE for each feature type. Specifically, these regression methods were GPR with a Matern kernel for both the SBM and VBM, and ridge regression for the similarity matrix features. The methods were picked by CV MAE instead of test MAE to prevent data leakage. Since the SBM and VBM features were not available for every image in the Icelandic dataset, we first calculated the average brain age prediction for each subject, before inner joining the predictions by subject into a single data frame. This resulted in a combined data frame with 1246 rows and three columns containing brain age predictions for the three regression methods. Since training these regression models is faster than training the CNNs, we were able to combine them using 10 -fold cross-validation predictions. Thus, the linear regression blender can train on predictions from the whole training set, instead of being limited to the 298 images in the validation set, as is the case for the CNN prediction combination.

Statistical methods. To assess the accuracy of the machine learning methods we performed simple training and validation splits, and selected a suitable model by evaluating the validation MAE. The subjects from the Icelandic sample were split between these three sets, and if a subject had multiple images, the images were all put in the same set. The data were divided into $64 \%$ training set $\left(N_{s}=809\right.$, $\left.N_{i}=1171\right), 16 \%$ validation set $\left(N_{s}=202, N_{i}=298\right)$, and $20 \%$ test set $\left(N_{s}=253\right.$, $N_{i}=346$ ), were $N_{s}$ is the number of subjects and $N_{i}$ is the number of images. When evaluating the machine learning models the MAE and $R^{2}$ score for the images in the validation and test set is calculated.

To assess the transfer learning performance, the IXI dataset was split into $80 \%$ training set $(N=440), 20 \%$ validation set $(N=104)$ and the whole UK Biobank dataset was used as a test set $(N=12395)$. As before, we evaluate accuracy by calculating the MAE and $R^{2}$ score on the validation and test set.

In order to test the reliability of PAD, the intraclass correlation was calculated with ICCbare from the ICC R package. The 95\% confidence interval was estimated using bootstrapping with 2000 sampling iterations.

The Pearson correlation coefficient was calculated in order to test for association between PAD and performance on neuropsychological tests. Before performing the association test we first removed individuals of non-white British ancestry and subjects from related pairs. We then adjusted the PAD for age at imaging visit, age $e^{2}$, sex, age $\times$ sex, age $e^{2} \times$ sex, total intracranial volume, the first 40 principal components from genetic ancestry analysis, head motion, genotyping array, imaging center, and assessment center where neuropsychological tests were conducted. The adjustments was performed using linear regression. Adjustment for variables such as genotyping array are probably not necessary for testing for association between PAD and performance on neuropsychological tests. However, we included them to keep the adjusted PAD similar to the one we perform the GWAS on. Nine correlation tests were performed, so a Bonferroni adjusted significance level $\alpha_{B 2}=0.05 / 9 \approx 0.00556$ was used. We estimated the $95 \%$ confidence interval using bootstrapping with 2000 sampling iterations.

We performed a GWAS on PAD using BOLT-LMM ${ }^{31}$ to find associated sequence variants. For the genetic analysis we used version 3 of the imputed genetic dataset released by UK Biobank in July $2017^{77}$. The UK Biobank genetic data were assayed using two very similar genotyping arrays ( $95 \%$ of marker content is shared). Roughly $10 \%$ of the subjects were genotyped using applied Biosystems UK BiLEVE Axiom Array by Affymetrix and the rest using the closely related Applied Biosystems UK Biobank Axiom Array ${ }^{77}$. Variants with imputation quality score below 0.3 , and minor allele frequency below $0.1 \%$ were filtered out, which left $\sim 20$ million variants to be considered for GWAS. Before performing GWAS, we removed individuals of non-white British ancestry and subjects from related pairs. We then adjusted the PAD for age at imaging visit, age ${ }^{2}$, sex, age $\times$ sex, age $e^{2} \times$ sex, total intracranial volume, the first 40 principal components from genetic ancestry analysis, head motion, genotyping array, and imaging center using linear regression. The adjusted PAD was then normalized with an inverse normal transformation. After normalization the linear regression adjustments were reapplied. Sequence variants associated with PAD are only reported if they reach genome-wide significance. If two genome-wide significant variants are in LD $\left(r^{2}>0.1\right)$ we report the variant with the lower $P$-value.

In addition, we tested for association between PAD and sequence variants known to associate with structural brain phenotypes. These variants were found by performing GWAS separately on 305 SBM phenotypes generated with recon-all by using the Freesurfer 6.0 software ${ }^{69}$ and 540 VBM phenotypes generated by using CAT12 ${ }^{56}$. All genome-wide significant markers were then aggregated into a single list. In cases where variants were in $\mathrm{LD}\left(r^{2}>0.5\right)$, only the variant with the lower $P$-value was selected. The final list included 331 variants, to account for testing test variants for the second time a Bonferroni adjusted significance level $\alpha_{B 3}=\frac{0.05}{2.331} \approx$ $7.5 \times 10^{-5}$ was used for the PAD association test.

To reduce the risk of false positive sequence variant associations we additionally checked for association in a replication set of 4456 subjects. To pass this test the association between the variants under consideration and PAD need to show evidence of statistical significance $\left(\alpha_{R}<0.05\right)$.

Heritability analysis. To estimate SNP-heritability $\left(h_{\text {snp }}^{2}\right)$ we ran LD score regres$\operatorname{sion}^{33}$ on the PAD GWAS summary statistics. We used the ldsc command line too and followed standard procedure when running it. To train the LD score regression model, we used precomputed European 1000 Genomes phase 3 LD Scores, and filtered out rare variants with $\mathrm{MAF}<0.01$ and imputation quality score $<0.9$. The slope of the trained regression model times the number of SNPs and divided by the sample size is an estimate of $h_{\mathrm{snp}}^{2}{ }^{33}$. In addition, the intercept of the model minus one is a measure of confounding bias in the test statistics due to confounding effect, such as cryptic relatedness and population stratification ${ }^{33}$.

eQTL analysis. To investigate if any of the variants are expression quantitative trait loci (eQTLs) we used the GTEx database (GTEx Analysis Release V7 [dbGaP Accession phs000424.v7.p2] $)^{78}$. Our eQTL analysis was carried out by logging onto https://gtexportal.org, typing in the corresponding rs number of identified variants, and checking if they have any associated eQTLs. However, identifying whether a variant is truly causal in both GWAS and eQTL is challenging because of the uncertainty caused by $\mathrm{LD}^{79}$. Therefore, we only report variants as eQTLs of genes if they are close to being the most significant $\mathrm{eQTL}$ of that specific gene.

Ethical regulations. The Icelandic participants in this study were recruited by deCODE genetics to study the cognitive and neurological effects of rare variants previously associated with schizophrenia and autism spectrum disorder. The UK Biobank oversaw the recruitment of subjects of British nationality. Approval for the aforementioned schizophrenia study was obtained from the National Bioethics Committee of Iceland and the Icelandic Data Protection Authority. Written informed consent was obtained from all participants or their guardians before blood samples or phenotypic data were obtained. All sample identifiers were encrypted in accordance with the regulations of the Icelandic Data Protection Authority. Information about ethics oversight in the UK Biobank can be found at https://www.ukbiobank.ac.uk/ethics/.

Reporting summary. Further information on research design is available in the Nature Research Reporting Summary linked to this article.

\section{Code availability}

Any custom code or software used to implement the brain age prediction method detailed in this paper will be made available upon request.

\section{Data availability}

The genetic and phenotype datasets generated by UK Biobank used in this study are available via the UK Biobank data access process (see http://www.ukbiobank.ac.uk/ register-apply/). Detailed information about the genetic data and MRI data available in UK Biobank is listed here: http://www.ukbiobank.ac.uk/scientists-3/genetic-data/, https:// www.fmrib.ox.ac.uk/ukbiobank/. The Icelandic data used in this publication are not publicly available due to information, contained within them, that could compromise research participant privacy. The authors declare that the data supporting the findings of this study are available within the article, its supplementary information, and upon request.

Received: 2 April 2019; Accepted: 21 October 2019; Published online: 27 November 2019

\section{References}

1. Cole, J. H. et al. Brain age predicts mortality. Mol. Psychiatry 23, 1385 (2018).

2. Abbott, A. A problem for our age. Nature 475, S2 (2011). 
3. Reeve, A., Simcox, E. \& Turnbull, D. Ageing and Parkinson's disease: why is advancing age the biggest risk factor? Ageing Res. Rev. 14, 19-30 (2014).

4. Franke, K., Ziegler, G., Klöppel, S. \& Gaser, C., Alzheimer's Disease Neuroimaging Initiative. et al. Estimating the age of healthy subjects from T1weighted MRI scans using kernel methods: exploring the influence of various parameters. Neuroimage 50, 883-892 (2010).

5. Liem, F. et al. Predicting brain-age from multimodal imaging data captures cognitive impairment. NeuroImage 148, 179-188 (2017).

6. Wang, J. Age estimation using cortical surface pattern combining thickness with curvatures. Med. Biol. Eng. Comput. 52, 331-341 (2014).

7. Kondo, C. et al. An age estimation method using brain local features for T1weighted images. In Engineering in Medicine and Biology Society (EMBC), 2015 37th Annual International Conference of the IEEE, 666-669 (IEEE, 2015).

8. Cole, J. H., Leech, R. \& Sharp, D. J., Alzheimer's Disease Neuroimaging Initiative. Prediction of brain age suggests accelerated atrophy after traumatic brain injury. Ann. Neurol. 77, 571-581 (2015).

9. LeCun, Y., Bengio, Y. \& Hinton, G. Deep learning. nature 521, 436 (2015).

10. LeCun, Y., Bottov, L., Bengio, Y. \& Haffner, P. Gradient-based learning applied to document recognition. Proc. IEEE 86, 2278-2324 (1998).

11. Huang, T.-W. et al. Age estimation from brain MRI images using deep learning. In Biomedical Imaging (ISBI 2017), 2017 IEEE 14th International Symposium on Biomedical Imaging, 849-852 (IEEE, 2017).

12. Cole, J. H. et al. Predicting brain age with deep learning from raw imaging data results in a reliable and heritable biomarker. NeuroImage 163, 115-124 (2017).

13. Franke, K., Luders, E., May, A., Wilke, M. \& Gaser, C. Brain maturation: predicting individual brainage in children and adolescents using structural MRI. NeuroImage 63, 1305-1312 (2012).

14. Gaser, C. et al. Brainage in mild cognitive impaired patients: predicting the conversion to Alzheimer's disease. PloS One 8, e67346 (2013).

15. Franke, K., Gaser, C., Manor, B. \& Novak, V. Advanced brainage in older adults with type 2 diabetes mellitus. Fronti. Aging Neurosci. 5, 90 (2013).

16. Koutsouleris, N. et al. Accelerated brain aging in schizophrenia and beyond: a neuroanatomical marker of psychiatric disorders. Schizophrenia Bull. 40, 1140-1153 (2013).

17. Schnack, H. G. et al. Accelerated brain aging in schizophrenia: a longitudinal pattern recognition study. Am. J. Psychiatry 173, 607-616 (2016).

18. Kuchinad, A. et al. Accelerated brain gray matter loss in fibromyalgia patients: premature aging of the brain? J. Neurosc. 27, 4004-4007 (2007).

19. Steffener, J. et al. Differences between chronological and brain age are related to education and self-reported physical activity. Neurobiol. Aging 40, 138-144 (2016).

20. Luders, E., Cherbuin, N. \& Gaser, C. Estimating brain age using highresolution pattern recognition: younger brains in long-term meditation practitioners. Neuroimage 134, 508-513 (2016).

21. Kaufmann, T. et al. Common brain disorders are associated with heritable patterns of apparent aging of the brain. Nat. Neurosci. 22, 1617-1623 (2019).

22. The Brainstorm Consortium, Anttila, V., Bulik-Sullivan, B., Finucane, H. K. \& Walter, R. K. Analysis of shared heritability in common disorders of the brain. Science 360, 6395 (2018).

23. Simonyan, K. \& Zisserman, A. Very deep convolutional networks for largescale image recognition. Preprint at http://arXiv.org/abs/1409.1556, (2014).

24. He, K., Zhang, X., Ren, S. \& Sun, J. Deep residual learning for image recognition. In Proceedings of the IEEE Conference on Computer Vision and Pattern Recognition, 770-778 (2016).

25. Li, H., Xu, Z., Taylor, G., Studer, C. \& Goldstein, T. Visualizing the loss landscape of neural nets. In Advances in Neural Information Processing Systems, 6389-6399 (2018).

26. Fischl, B. \& Dale, A. M. Measuring the thickness of the human cerebral cortex from magnetic resonance images. Proc. Natl Acad.Sci. USA 97, 11050-11055 (2000).

27. Ashburner, John \& Friston, K. J. Voxel-based morphometry-the methods. Neuroimage 11, 805-821 (2000).

28. Tipping, M. E. The relevance vector machine. In Advances in Neural Information Processing Systems, 652-658 (2000).

29. Rasmussen, C. E. Gaussian processes in machine learning. In Advanced Lectures on Machine Learning, 63-71 (Springer, 2004).

30. Sudlow, C. et al. Uk Biobank: an open access resource for identifying the causes of a wide range of complex diseases of middle and old age. PLoS Med. 12, e1001779 (2015)

31. George, P.-R. et al. Efficient bayesian mixed-model analysis increases association power in large cohorts. Nat. Genet. 47, 284 (2015).

32. Smith, S. M. \& Nichols, T. E. Statistical challenges in "big data" human neuroimaging. Neuron 97, 263-268 (2018).

33. Bulik-Sullivan, B. K. et al. Ld score regression distinguishes confounding from polygenicity in genome-wide association studies. Nat. Genet. 47, 291 (2015).

34. Stefansson, H. et al. A common inversion under selection in europeans. Nat. Genet. 37, 129-137 (2005).
35. Rademakers, R., Cruts, M. \& Van Broeckhoven, C. The role of tau (MAPT) in frontotemporal dementia and related tauopathies. Hum. Mut. 24, 277-295 (2004).

36. Koolen, D. A. et al. A new chromosome 17q21. 31 microdeletion syndrome associated with a common inversion polymorphism. Nat. Genet. 38, 999 (2006).

37. Nagel, M., Jansen, P. R. \& Stringer, S. et al. Meta-analysis of genome-wide association studies for neuroticism in 449484 individuals identify novel genetic loci and pathways. Nat. Genet. 50, 920-927 (2018).

38. Kong, A. et al. Selection against variants in the genome associated with educational attainment. Proc. Natl Acad. Sci USA. 114, E727-E732 (2017).

39. Ikram, M. A., Fornage, M. \& Smith, A. V. et al. Common variants at $6 \mathrm{q} 22$ and $17 \mathrm{q} 21$ are associated with intracranial volume. Nat. Genet. 44, 539-544 (2013).

40. Nalls, M. A. et al. A meta-analysis of genome-wide association studies identifies 17 new Parkinson's disease risk loci. Nat. Genet. 49, 1511-1516 (2017).

41. Riccelli, R., Toschi, N., Nigro, S., Terracciano, A. \& Passamonti, L. Surfacebased morphometry reveals the neuroanatomical basis of the five-factor model of personality. Soc. Cogn. and Affect. Neurosci. 12, 671-684 (2017).

42. Hervieu, G. J. et al. Distribution and expression of TREK-1, a two-poredomain potassium channel, in the adult rat CNS. Neuroscience 103, 899-919 (2001).

43. Bittner, S., Ruck, T., Fernández-Orth, J. \& Meuth, S. G. Trekking the blood-brain-barrier. J. Neuroimm. Pharmacol. 9, 293-301 (2014).

44. Cai, Y., Peng, Z., Guo, H., Wang, F. \& Zeng, Y. TREK-1 pathway mediates isoflurane-induced memory impairment in middle-aged mice. Neurobiol. Learn. Mem. 145, 199-204 (2017).

45. Wang, W. et al. Lig4-4 selectively inhibits TREK-1 and plays potent neuroprotective roles in vitro and in rat mcao model. Neurosci. Lett. 671, 93-98 (2018)

46. Guen, Y. L. et al. eQTL of KCNK2 regionally influences the brain sulcal widening: evidence from 15,597 UK Biobank participants with neuroimaging data. Brain Struct. Funct. 224, 847-857 (2018)

47. Stein, G. S. et al. Runx 2 control of organization, assembly and activity of the regulatory machinery for skeletal gene expression. Oncogene 23, 4315 (2004)

48. Nagy, R. et al. Exploration of haplotype research consortium imputation for genome-wide association studies in 20,032 generation scotland participants. Gen. Med. 9, 23 (2017).

49. Lee, J. J. et al. Gene discovery and polygenic prediction from a genome-wide association study of educational attainment in 1.1 million individuals. Nat. Genet. 50, 1112 (2018).

50. Pirastu, N. et al. Gwas for male-pattern baldness identifies 71 susceptibility loci explaining 38\% of the risk. Nat. Commun. 8, 1584 (2017).

51. Reuter, M. et al. Head motion during MRI acquisition reduces gray matter volume and thickness estimates. Neuroimage 107, 107-115 (2015).

52. Elliott, L. T. et al. Genome-wide association studies of brain imaging phenotypes in UK Biobank. Nature 562, 210 (2018).

53. Salthouse, T. A. What cognitive abilities are involved in trail-making performance? Intelligence 39, 222-232 (2011).

54. Deary, I. J. et al. Age-associated cognitive decline. Br. Med. Bullet. 92, 135-152 (2009).

55. Marchini, J., Cardon, L. R., Phillips, M. S. \& Donnelly, P. The effects of human population structure on large genetic association studies. Nat. Genet. 36, 512 (2004).

56. Gaser, C. \& Dahnke, R. Cat-a computational anatomy toolbox for the analysis of structural MRI data. HBM 2016, 336-348 (2016).

57. Ashburner, J. A fast diffeomorphic image registration algorithm. Neuroimage 38, 95-113 (2007).

58. Kurth, F. \& Gaser, C. Manual - Computational Anatomy Toolbox - CAT12 (2017).

59. Chollet, F. et al. Keras. https://keras.io (2015)

60. Ioffe, S. Batch renormalization: Towards reducing minibatch dependence in batch-normalized models. In Advances in Neural Information Processing Systems, 1945-1953 (2017).

61. Clevert, D.-A., Unterthiner, T. \& Hochreiter, S. Fast and accurate deep network learning by exponential linear units (elus). Preprint at http://arXiv. org/abs/1511.07289, (2015).

62. Zell, A. Simulation Neuronaler Netze, volume 1 (Addison-Wesley, Bonn, 1994).

63. Srivastava, N., Hinton, G., Krizhevsky, A., Sutskever, I. \& Salakhutdinov, R. Dropout: a simple way to prevent neural networks from overfitting. J. Mach. Learn. Res. 15, 1929-1958 (2014)

64. Kingma, D. P. \& Ba, J. Adam: a method for stochastic optimization. Preprint at http://arXiv.org/abs/1412.6980, (2014).

65. He, K., Zhang, X., Ren, S. \& Sun, J. Delving deep into rectifiers: surpassing human-level performance on imagenet classification. in Proceedings of the IEEE International Conference on Computer Vision, 1026-1034 (2015). 
66. Krogh, A. \& Hertz, J. A. A simple weight decay can improve generalization. In Advances in Neural Information Processing Systems, 950-957 (1992).

67. Morgan, N. \& Bourlard, H. Generalization and parameter estimation in feedforward nets: Some experiments. In Advances in Neural Information Processing Systems, 630-637 (1990).

68. Goodfellow, I., Bengio, Y., Courville, A. \& Bengio, Y. Deep Learning: Data Augmentation, volume 1 (MIT press Cambridge, 2016)

69. Fischl, B. Freesurfer. Neuroimage 62, 774-781 (2012).

70. Seber, G. A. F. \& Lee, A. J. Linear Regression Analysis, volume 329. (John Wiley \& Sons, 2012).

71. Tibshirani, R. Regression shrinkage and selection via the lasso. J. R. Stat. Soc. B 58, 267-288 (1996).

72. Hoerl, A. E. \& Kennard, R. W. Ridge regression: biased estimation for nonorthogonal problems. Technometrics 12, 55-67 (1970).

73. Zou, H. \& Hastie, T. Regularization and variable selection via the elastic net. $J$. R. Stat. Soc. B 67, 301-320 (2005).

74. Ho, T. K. Random decision forests. In Document Analysis and Recognition, Proceedings of the Third International Conference on Document Analysis and Recognition, volume 1, 278-282 (IEEE, 1995).

75. Smola, A. J. \& Schölkopf, B. A tutorial on support vector regression. Stat. Comput. 14, 199-222 (2004).

76. Pedregosa, F. et al. Scikit-learn: machine learning in Python. J. Mach. Learn. Res. 12, 2825-2830 (2011).

77. Bycroft, C. et al. The UK Biobank resource with deep phenotyping and genomic data. Nature 562, 203 (2018).

78. GTEx Consortium. et al. Genetic effects on gene expression across human tissues. Nature 550, 204 (2017).

79. Hormozdiari, F. et al. Colocalization of GWAS and eQTL signals detects target genes. Am. J. Hum. Genet. 99, 1245-1260 (2016).

\section{Acknowledgements}

This research has been conducted using the UK Biobank Resource under Application Number 24898. The research leading to these results has received support from the Innovative Medicines Initiative Joint Undertaking under grant agreements no. 115008 (NEWMEDS) and no. 115300 (EUAIMS), of which resources are composed of EFPIA inkind contribution and financial contribution from the European Union's Seventh Framework Programme (EU-FP7/2007-2013). The financial support from the European Commission to the NeuroPain project (FP7\#HEALTH-2013-602891-2) is acknowledged. The authors are grateful to the participants, and we thank the research nurses and staff at the Recruitment centre (Pjónustumiðstöð rannsóknarverkefna).

\section{Author contributions}

B.A.J. implemented the method, wrote the code, and performed experiments. B.A.J. and M.O.U. developed the method and designed statistical experiments. G.B., T.T., G.B.W., L.M.E., D.F.G., H.S., and K.S. contributed to analyses of the data and writing the manuscript.

\section{Competing interests}

B.A.J., G.B., T.T., G.B.W., D.F.G., H.S., K.S., and M.O.U. are employed by deCODE genetics/Amgen, Inc. L.M.E. declares no competing interests.

\section{Additional information}

Supplementary information is available for this paper at https://doi.org/10.1038/s41467 019-13163-9.

Correspondence and requests for materials should be addressed to K.S. or M.O.U.

Peer review information Nature Communications thanks James Cole and the other, anonymous, reviewers for their contribution to the peer review of this work. Peer reviewer reports are available.

Reprints and permission information is available at http://www.nature.com/reprints

Publisher's note Springer Nature remains neutral with regard to jurisdictional claims in published maps and institutional affiliations.

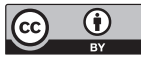

Open Access This article is licensed under a Creative Commons Attribution 4.0 International License, which permits use, sharing, adaptation, distribution and reproduction in any medium or format, as long as you give appropriate credit to the original author(s) and the source, provide a link to the Creative Commons license, and indicate if changes were made. The images or other third party material in this article are included in the article's Creative Commons license, unless indicated otherwise in a credit line to the material. If material is not included in the article's Creative Commons license and your intended use is not permitted by statutory regulation or exceeds the permitted use, you will need to obtain permission directly from the copyright holder. To view a copy of this license, visit http://creativecommons.org/ licenses/by/4.0/.

(C) The Author(s) 2019 